\title{
DIABETIC NEUROPATHY
}

natuire DISEASE

REVIEWS PRIMERS

Diabetic neuropathy is the most common complication of diabetes mellitus. Neuropathy can manifest in various ways, although the most common presentation is distal symmetric polyneuropathy, whereby the hands and polyneu lower limbs are predominantly affected.

\section{EPIDEMIOLOGY}

The annual cost of diabetic neuropathy is more than $\$ 10$ billion in the United States
The incidence of diabetic neuropathy is 6,100 per 100,000 person-years in patients with type 2 diabetes mellitus (T2DM) and 2,800 per 100,000 person-years in patients with type 1 diabetes mellitus (T1DM)

Symptoms of diabetic neuropathy include numbness, tingling, pain and unsteadiness in a 'stocking and glove' distribution. Clinical findings are the loss of sensation for pinprick, temperature and vibration, in N addition to loss (assessed by small movements of the great toe). Objective evidence of loss of sensation (using, for example, nerve conduction studies) is not required in routine clinical practice and is generally used only in research studies.

\section{Ci) OUTLOOK}

Diabetic neuropathy remains the only microvascular complication of diabet mellitus that lacks a specific treatment. the number of cases of diabetes mellitus and obesity continues to increase, new therapies for diabetic neuropathy are urgently required.

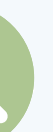

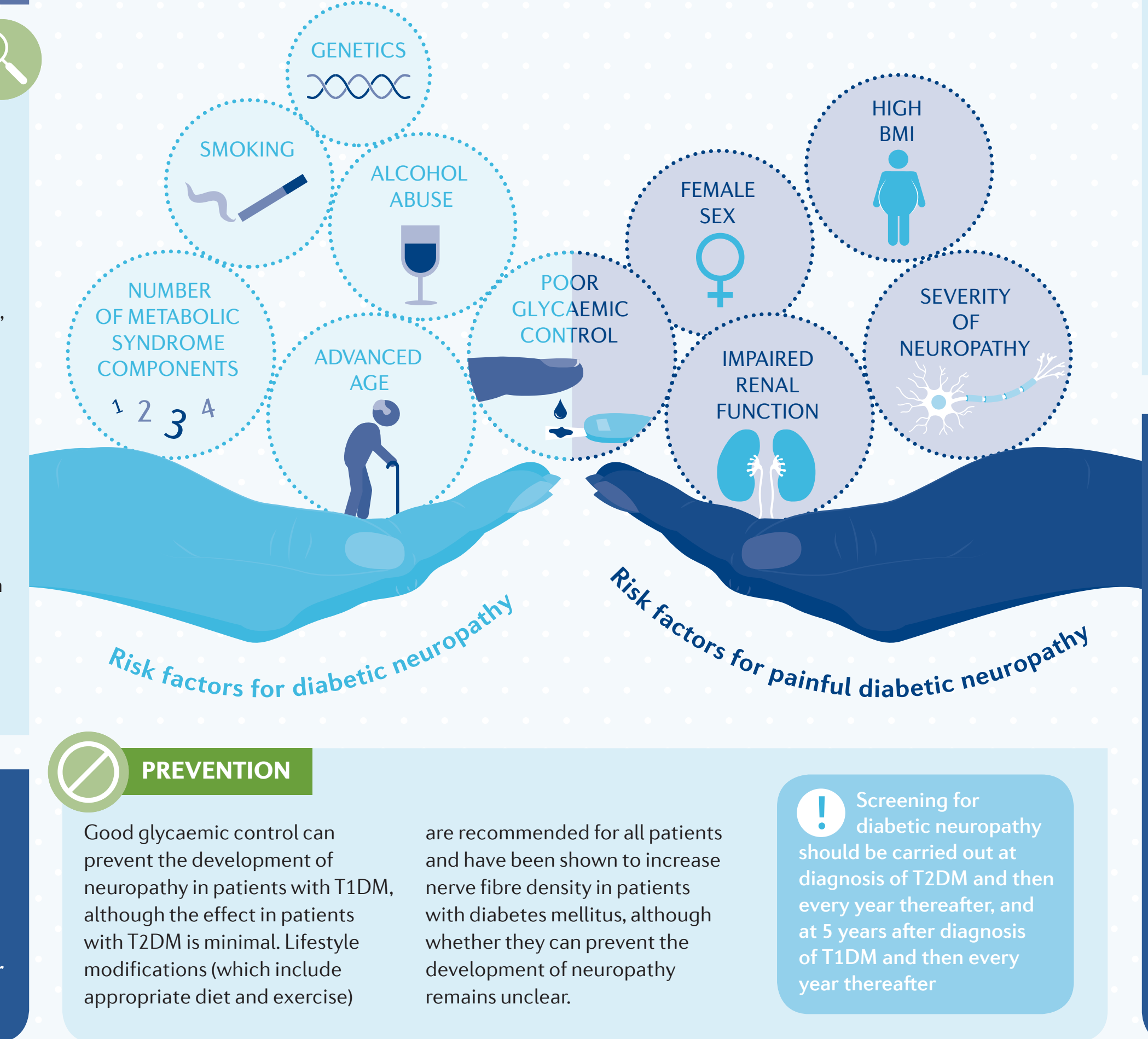

For the Primer, visit doi:101038/s41572-019-0092-1

\section{MECHANISMS}

Hyperglycaemia and dyslipidaemia lead to several pathological alterations in glucose and lipid metabolism that can affect neuronal function and contribute to diabetic neuropathy through reactive oxygen species (ROS) production. Hyperglycaemia also contributes to the production of advanced glycation end products (AGEs), whereby reactive carbohydrate groups are added to cellular proteins and lipids, leading to altered function. AGEs and oxidised LDL can contribute to inflammatory signals, whereas dysregulated insulin signalling leads to a loss of neurotrophic signalling. The precise cellular response to hyperglycaemia or hyperlipidaemia depends on the affected cell type. Although the entire neuronal tree is affected in diabetic neuropathy, whether damage initially targets the axon and associated Schwann cell, or the neuronal cell body is unknown.

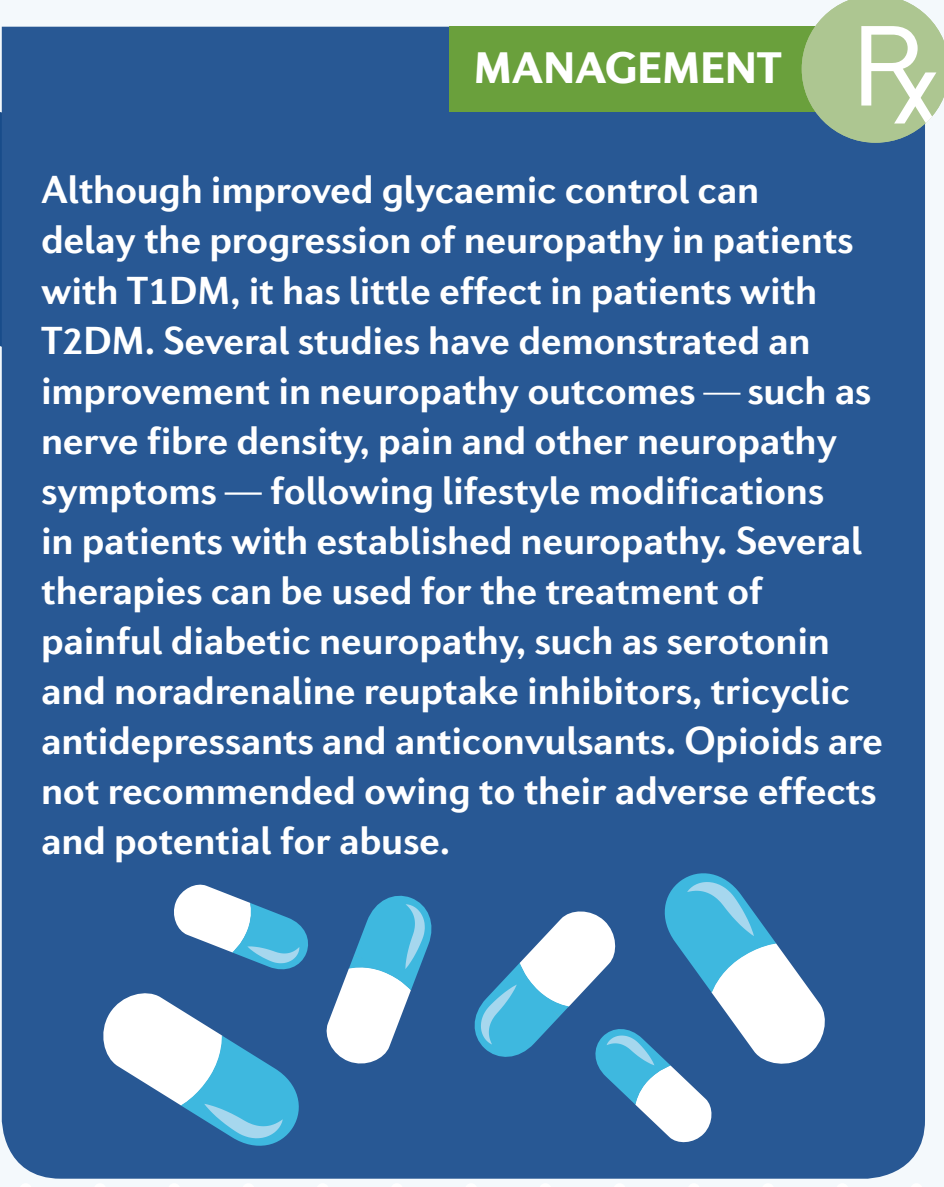

